\title{
Commentary: Purpuric skin eruption in an Illicit drug User: Levamisole-Induced vasculitis
}

\author{
Kristen Whitworth*, Nicholas Graff, and Christopher Trigger \\ Lakeland Health Emergency Medicine Residency Program, 1234 Napier Avenue, St. Joseph, MI 49085, USA
}

Article Info

\section{Article Notes}

Received: April 19, 2017

Accepted: May 23, 2017

\section{${ }^{*}$ Correspondence:}

Kristen Whitworth, DO, Lakeland health emergency medicine residency program, 1234 napier avenue, St. Joseph, MI 49085, USA, E-mail: kwhitworth1@lakelandhealth.org

(C) 2017 Kristen Whitworth. Cavalieri. This article is distributed under the terms of the Creative Commons Attribution 4.0 International License.
The first case connecting vasculitis to levamisole adulterated cocaine was published in 2010. Since that time, there have been numerous case reports of complications arising from levamisole contaminated cocaine. A case report in 2011 suggested the use of the term LINES to describe the levamisole induced syndrome of vasculitis, neutropenia, and purpura resulting in skin necrosis ${ }^{1}$. The illustrative acronym LINES - levamisole induced necrosis syndrome - was coined as a tongue-and-check reference to the manner cocaine is dosed when used illicitly. While the community is developing a better understanding of this syndrome, other potential complications of levamisole are coming into light.

In the past 7 years, we have gained more understanding of complications related to levamisole adulterated cocaine. As we are aware, levamisole adulterated cocaine is associated with an antineutrophil cytoplasmic antibody-associated (ANCA) vasculitis, notable for its cutaneous involvement ${ }^{2}$. There are, however, still many questions that remain unanswered. The exact pathophysiology of the development of agranulocytosis from levamisole is unknown. Levamisole is an immunostimulant whose greatest mechanism of action is increasing monocyte chemotaxis ${ }^{3}$. It also enhances macrophage function, promotes maturation of $\mathrm{T}$ cells and T lymphocytes, and increases cytokine production ${ }^{4}$. These actions create an inflammatory response which may contribute to the development of agranulocytosis. We are also still unsure why certain people appear to at increased risk to developing LINES or if there is a true toxic dose of levamisole for any organ system. Doses of $0.36 \mathrm{mg} /$ day were not notably toxic in animal and human studies, but further information concerning the trials is required to confirm this data ${ }^{5}$. Some assumptions can be made from published studies regarding levamisole when it was used as immunomodulatory drug prior to being withdrawn from the European market, United States and Canada 1999. Episodes of agranulocytosis after levamisole exposure was found to be more frequent among females in one study ${ }^{6}$. The cause for an increased incidence in women is unknown, however one possible explanation could be that females have quicker oral absorption of levamisole than males. ${ }^{6}$ In another study, patients with the HLA B27 genotype were found to have a higher incidence of agranulocytosis ${ }^{6}$. From these published case reports, it is evident that after an initial episode of levamisole induced vasculitis, patients have a lower threshold for a recurrent episode with repeat exposure.

There have been case reports linking levamisole and levamisole 
adulterated cocaine with leukoencephalopathy, as well as membranous nephropathy and pulmonary vasculitis. In 2005, the World Health Organization published recommendations to remove levamisole from their list of Essential Medicines after a decade's worth of reports from China emerged detailing 543 case reports of levamisole-induced demyelinating encephalopathy ${ }^{7}$. As recent as 2015 there have been case reports correlating levamisole exposure with crescentic rapidly progressive glomerulonephritis due to its propensity to induce an ANCA-associated vasculitis ${ }^{8,9}$. There are two case reports describing autopsy findings of pulmonary lymphocytic vasculitis in illicit drug users with known levamisole exposure. It is unclear if this contributed to their death or was an incidental postmortem finding ${ }^{10}$. An analysis of patients with newly diagnosed ANCA-positive systemic disease associated cocaine use around the year 2010 found there were three patients with pulmonary hemorrhage ${ }^{2}$. Long term complications from an episode of levamisole induced vasculitis are not well defined in the literature, but it can be assumed that complications would pertain to the extent and location of vasculitis. We should also consider that some idiopathic vasculitic cases could potentially be the adverse effects of long term exposure to levamisole, especially in chronic cocaine users that never present with an acute illness, or patients that are unknown to use illicit substances. With the expanding literature linking these complications with levamisole induced vasculitis, additional cases may come to light as physicians are becoming more aware of these complications and should remain vigilant in at-risk patient populations.

Following the initial publication of this case report, we performed further investigation into our patient's hospital stay after transfer to a tertiary center secondary to extensive cutaneous losses on both of her proximal lower extremities requiring a burn specialist. She was noted to have an episode of hemoptysis while being cared for at a tertiary center which prompted a CT angiogram of her chest. She was found to have dense consolidation of her right lower lobe, consistent with necrosis, and with a small reactive pleural effusion, and notable intrathoracic adenopathy. Unfortunately, from an academic aspect, no bronchoscopy was performed to confirm the potential diagnosis of pulmonary lymphocytic vasculitis. Prior to being lost to follow up, our patient had recurrent nonhealing ulcerations to her lower extremities for which she was being followed by a general surgeon at our local chronic wound clinic. It is unclear if her episode of levamisole induced vasculitis was contributory to these ulcerations, as she had a history of previous lower extremity ulcerations as well as previous MRSA infections.

There is still no standard of care for treatment of levamisole induced vasculitis. The characteristic skin lesions can range from purpura to full thickness necrosis.
These lesions are treated similar to burns with wound care dressings and wound debridement with indications for antibiotics only if clinical evidence of infection. There is still no definitive stance on the role for prophylactic antibiotics or steroids. As these vasculitic lesions often coincide with neutropenia and agranulocytosis, they are at a greater risk for infection. Depending on the severity of the wounds, skin grafting or amputation may be required. The predilection of the nasal and auricular necrosis in this syndrome further complicates treatment as these cartilaginous regions have poorer healing and carry high cosmetic value. When these regions are involved, plastic surgery should be involved for optimal cosmesis.

While levamisole induced vasculitis and its associated complications are becoming more common, it is still a problematic diagnosis for physicians to make. The presence of levamisole may be tested for in both the plasma and the urine, however a negative test dose not rule out the possibility as it has a short plasma elimination half-life. If there is clinical suspicion based on a history of cocaine abuse, exposure to levamisole should be assumed based on its increasing prevalence as a contaminate in cocaine. Another option would be to test for the presence of levamisole in hair samples. It is also important to keep other processes of cutaneous necrosis in mind during the initial workup, even if there is a strong suspicion for levamisole induced vasculitis. The addition of ANCA, cryoglobulins and antiphospholipid antibodies can aid in differentiating levamisole induced vasculitis from other potential causes. Dermal biopsies should be considered in most cutaneous pathology, especially if it is undifferentiated or there is potential for specific therapeutic management.

The rationale of cutting cocaine with levamisole is multifactorial. It is speculated that Levamisole is incorporated into cocaine for its potentiating effects. Levamisole has been found to inhibit monoamine oxidase $^{11}$. This combined with its nicotinic receptor agonist activity could potentially increase dopamine transmission in the brain. Additionally, levamisole is inexpensive, and therefore increases profit margins when used to dilute cocaine. Levamisole is easily obtained as well as difficult to detect at the street level when added to cocaine.

We as practitioners also need to keep in mind that levamisole is not the only compound being added to cocaine. Many other substances including caffeine, ephedrine, ketamine, and acetaminophen are being added to cocaine to increase profit margins. Benzocaine and phenacetine are often added for the classic "numbing effect" of cocaine which aids in further disguising additives. This is alarming as the benzocaine being used is not regulated by the FDA or any other regulating body. The use of phenacetine is also alarming as it was withdrawn from the US market by the FDA in 1983 after it was linked to renal failure and was 
found to be carcinogenic. These compounds may have side effects that interact with levamisole, potentially causing more adverse side effects. Many users have no idea what adulterants are in the cocaine they have purchased, let alone the dosing of these substances.

In closing, cutaneous vasculitis can be a devastating and deadly process and in the case of levamisole induced vasculitis can lead to multi-organ injury. While the Drug Enforcement Agency recently confirmed decreasing rates of levamisole within confiscated cocaine, an investigative report from Vice Media in 2015 brought to light the possibility of 40 to $90 \%$ of street cocaine to be cut with up to $5 \%$ levamisole ${ }^{5,12}$. This is also a preventable process and more information should be gained concerning the incidence of cocaine and other illicit drugs cut with levamisole in order to avoid exposure and prevent this vasculitic process.

\section{References}

1. Mouzakis J, Somboonwit C, Lakshmi S, et al. Levamisole Induced Necrosis Of The Skin And Neutropenia Following Intranasal Cocaine Use: A Newly Recognized Syndrome. Journal of Drugs in Dermatology. 2017; 10.10: 1204-7.

2. McGrath M, Isakova T, Rennke HG, et al. Contaminated Cocaine And Antineutrophil Cytoplasmic Antibody-Associated Disease. Clinical Journal of the American Society of Nephrology. 2011; 6.12: 2799-2805.

3. Scheinfeld N, Rosenberg J, Weinberg J. Levamisole in Dermatology. American Journal of Clinical Dermatology. 2004; 5(2): 97-104.
4. Szeto C, Gillespie K, Mathieson P. Levamisole induces interleukin-18 and shifts type 1 /type 2 cytokine balance. Immunology. 2000; 100(2): 217-224.

5. Drug Enforcement Administration. Levamisole (Ergamisol).; 2013. Available at: http://www.deadiversion.usdoj.gov/drug_chem_info/ levamisole.pdf

6. Brunt T, van den Berg J, Pennings E, et al. Adverse Effects Of Levamisole In Cocaine Users: A Review And Risk Assessment. Archives of Toxicology. 2017; 1947-4.

7. Recommendations of The Third Meeting Of The WHO Advisory Committee On Safety Of Medicinal Products. N.p., 2005. Web. 19 Apr. 2017

8. Álvarez Díaz H, Marińo Callejo AI, García Rodríguez JF, et al. ANCApositive vasculitis induced by levamisole-adulterated cocaine and nephrotic syndrome: The kidney as an unusual target. American Journal of Case Reports. 2013; 14: 557-561.

9. Moinuddin I, Madhrira M, Bracamonte E, et al. Membranous Nephropathy With Crescents Associated With Levamisole-Induced MPO-ANCA Vasculitis. Pathology - Research and Practice. 2016; 212.7: 650-653.

10. Karch S, Busardò FP, Vaiano F, et al. Levamisole Adulterated Cocaine And Pulmonary Vasculitis: Presentation Of Two Lethal Cases And Brief Literature Review. Forensic Science International. 2017; 265: 96-102.

11. Agarwal A, Shukla O, Ghatak S, et al. Biogenic amines, metabolites and monoamine oxidase in the filarial worm Setaria cervi. International Journal for Parasitology. 1990; 20(7): 873-881. doi:10.1016/00207519(90)90025-i.

12. Daly M. The Truth About Britain's 'Flesh-Eating' Cocaine. Vice Np. 2015. 